\title{
SEROLOGICAL MARKERS FOR METASTATIC BREAST CANCER
}

\author{
J.S.Y. NG*, C.M. STURGEON $\$$, J. SETH ${ }^{\$}$, G.M. PATERSON\$, J.E. ROULSTON\$, \\ R.C.F. LEONARD*† \\ *Departments of Clinical Oncology, Western General Hospital, Edinburgh EH4 $2 X U$ \\ ${ }^{\$}$ Clinical Biochemistry, Royal Infirmary, Edinburgh EH3 9YW, U.K. \\ On behalf of the Edinburgh Breast Group \\ (U. Chett), J.M. Dixon, R.C.F. Leonard, L.M. Matheson and I.H. Kunkler).
}

\begin{abstract}
SUMMARY
Three serum markers, TPS, CA 15.3 and CEA, were used to monitor the response to treatment of 20 patients with metastatic breast cancer. At the time of the first evidence of metastases or at the time of progression of known metastatic disease, $84 \%$ of TPS values were above the reference limit, as compared to $74 \%$ for CA 15.3 and $84 \%$ for CEA. If the treatment instituted was effective, $60 \%$ of TPS values showed an early (within 2 or 3 weeks after commencement or change of therapy) reduction in level against only $27 \%$ of CA 15.3 and $27 \%$ of CEA levels. This suggests that TPS provides a more sensitive and earlier predictor of therapeutic response.

In patients with clinical evidence of further progression of disease while on therapy, $86 \%$ of TPS values showed persistent elevation or increase, as compared to $71 \%$ of CA 15.3 levels and only $36 \%$ of CEA levels. It was also noted in these patients that TPS values rose earlier than either CA 15.3 or CEA. This indicates that TPS is a more reliable predictor of response to treatment than the other two markers.

In addition, we found that, at the time of presentation, in women who had visceral metastases (liver, lung, or brain alone or in combination), $87 \%$ of TPS values were raised, as compared to $80 \%$ of CA 15.3 and $73 \%$ of CEA values. In women who had bone and soft tissue metastases at presentation, $75 \%$ of TPS values were elevated, against $50 \%$ of CA 15.3 and $75 \%$ of CEA values. We also noted that in patients without raised TPS levels, the sites of subsequent disease progression were limited to bones, regional lymph nodes, skin and soft tissues.
\end{abstract}

KEY WORDS Breast cancer Metastases Serological markers

\section{INTRODUCTION}

In the post-operative follow-up of breast cancer, serological tumour markers are noted to be useful in the early detection and monitoring of metastases (Haagensen, 1982; Bieglmayer et al., 1988; Merimsky et al., 1991). The markers enable relapses to be treated early, and in responding women may, with effective treatment, significantly increase the duration of disease-free and sometimes overall survival (Merimsky et al., 1991; Jager et al., 1992). Serial measurements of serological markers may allow a reduction in the number of radiological examinations (Nicolini et al., 1989, 1991) and further increase the cost effectiveness ratio of palliative treatment.

Correspondence to: R.C.F. Leonard, Department of Clinical Oncology, Western General Hospital, Edinburgh EH4 2XU, U.K. 
A number of tumour markers have been used to monitor metastatic breast cancer, CA 15.3 and CEA probably being the most widely studied. While these markers are generally thought to reflect tumour bulk, tissue polypeptide antigen (for which a specific antigenic determinant, known as TPS, can now be measured) is claimed to be an indicator of cell proliferation activity, and may give different information. Comparison of the usefulness of these markers in breast cancer patients is of considerable interest (Bjorklund, 1992; van Dalen, 1992). In a cohort of 20 patients receiving treatment for metastatic breast cancer, we have, therefore, measured the markers in order to evaluate three parameters: (i) frequency of expression of the markers, (ii) their sensitivity, and (iii) their accuracy in reflecting clinical response. We report here local experience with these markers, the results also forming part of a multicentre trial (van Dalen et al., 1993).

\section{MATERIALS AND METHODS}

\section{Breast cancer patients}

We have examined 20 women either with first evidence of metastases outwith the local area, or with clinical evidence of progression of known metastatic disease requiring change of treatment. Tumour pathology included carcinoma of no special histological sub-type (50\%), ductal carcinoma (35\%), lobular carcinoma (5\%), scirrhous carcinoma $(5 \%)$ and mucoid carcinoma $(5 \%)$. Assessment of response to therapy was made at 3 and 6 months after commencement or change of treatment and was according to conventional UICC criteria using clinical and radiological techniques.

\section{Blood collection}

Blood was collected from patients in the study before commencement or change of therapy and then at 2 weeks, 1, 2, 3, 4 and 6 months thereafter. Following separation from the clot, serum was divided into three aliquots and stored at $-30^{\circ} \mathrm{C}$ prior to measurement of tumour markers.

\section{Assay methods}

Commercially available immunoradiometric assays (IRMA) were employed for measurement of TPS (TPS IRMA, Beki Diagnostics AB, Bromma, Sweden) and CA 15.3 (CA15.3 ELSA, CIS Ltd, High Wycombe, U.K.). Assays were performed according to the manufacturers' instructions. CEA was measured by immunoradiometric assay, using monoclonal antibodies to CEA (Scottish Antibody Production Unit, Carluke, Scotland). The assay is calibrated in U/L of the international reference preparation for CEA (IRP 73/ 601) (Laurence et al, 1975). (In this assay, $11 \mathrm{U} / \mathrm{L}$ is approximately equivalent to $1 \mathrm{ng} /$ $\mathrm{mL}$ of CEA). Appropriate quality control samples were included in each assay batch and between-batch precision was satisfactory (coefficients of variation $<10 \%$ ). Previously established reference ranges were used to evaluate results ( $95 \%$ confidence limits for normal blood donors: TPS, $80 \mathrm{U} / \mathrm{L}$; CA 15.3, $35 \mathrm{u} / \mathrm{mL}$; and CEA, $60 \mathrm{U} / \mathrm{L}$ ). Changes in marker level of less than $15 \%$ were regarded as insignificant (ie levels were considered as remaining stable). 
Table 1. Number of patients with elevated marker results (TPS, CA 15.3, and CEA) at the time of first evidence of metastases or the time of progression of known metastatic disease.

\begin{tabular}{c|c}
\hline Marker & $\begin{array}{r}\text { No. (\%) of patients with elevated markers at the time } \\
\text { of first evidence of metastases or the time of } \\
\text { progression of known metastatic disease }\end{array}$ \\
\hline TPS & $16(84 \%)$ \\
CA 15.3 & $14(74 \%)$ \\
\hline
\end{tabular}

\section{RESULTS AND DISCUSSION}

In our study, at the time of first evidence of metastases of breast cancer, or the time of progression of known metastatic disease, $84 \%$ of TPS levels expressed were above the reference limit, as compared to $74 \%$ of CA 15.3 levels and $84 \%$ of CEA levels (Table I).

After commencement or change of therapy, and if the treatment instituted was effective, as denoted by at least a partial remission (UICC), $60 \%$ of TPS values fell within the first 2 or 3 weeks after commencement of such effective therapy, as illustrated by serial marker results for Patient HF (Fig. 1). This was compared against only $27 \%$ of elevated CA 15.3 values and $27 \%$ of elevated CEA values, showing similar early reductions (Table 2). In partial remission, only $8 \%$ of TPS values showed an unexpected elevation or rising trend, whereas $17 \%$ of CA 15.3 and $17 \%$ of CEA values showed misleading trends. The median lead time for TPS in these responses was 9 weeks (range 8-10 weeks), compared with medians of 8 weeks for CA 15.3 (range 6-10 weeks) and CEA (range 6-9 weeks). This indicates TPS is a more sensitive and possibly earlier predictor of the effectiveness of therapy.

Among patients with progressive disease while on therapy, $86 \%$ of TPS values were persistently raised or increasing, as compared to $71 \%$ of CA 15.3 and only $36 \%$ of CEA levels (Table 3). In patients with progressive disease, only 14\% of TPS values showed a falling trend out of accord with the clinical picture, as compared to $29 \%$ of CA 15.3 values and $57 \%$ of CEA values which showed misleading trends. Elevated or rising TPS levels were observed before corresponding levels of the other markers started to rise, as illustrated by results for Patient EN (Fig. 2). The median lead time for TPS in these disease progressions was 8 weeks (range 3-10 weeks), again greater than that of either CA 15.3 (median 5 weeks; range 0-10 weeks) or CEA (median 5 weeks; range 4-10 weeks), suggesting that TPS is a more sensitive and early detector of further progression of metastatic disease while the patient is on therapy.

In the assessment of longer term effects of therapy, however, we may need to take into consideration CA 15.3 and/or CEA values as well as TPS. This is because, in a "sustained partial remission", only $67 \%$ of elevated TPS levels showed a falling trend, compared 


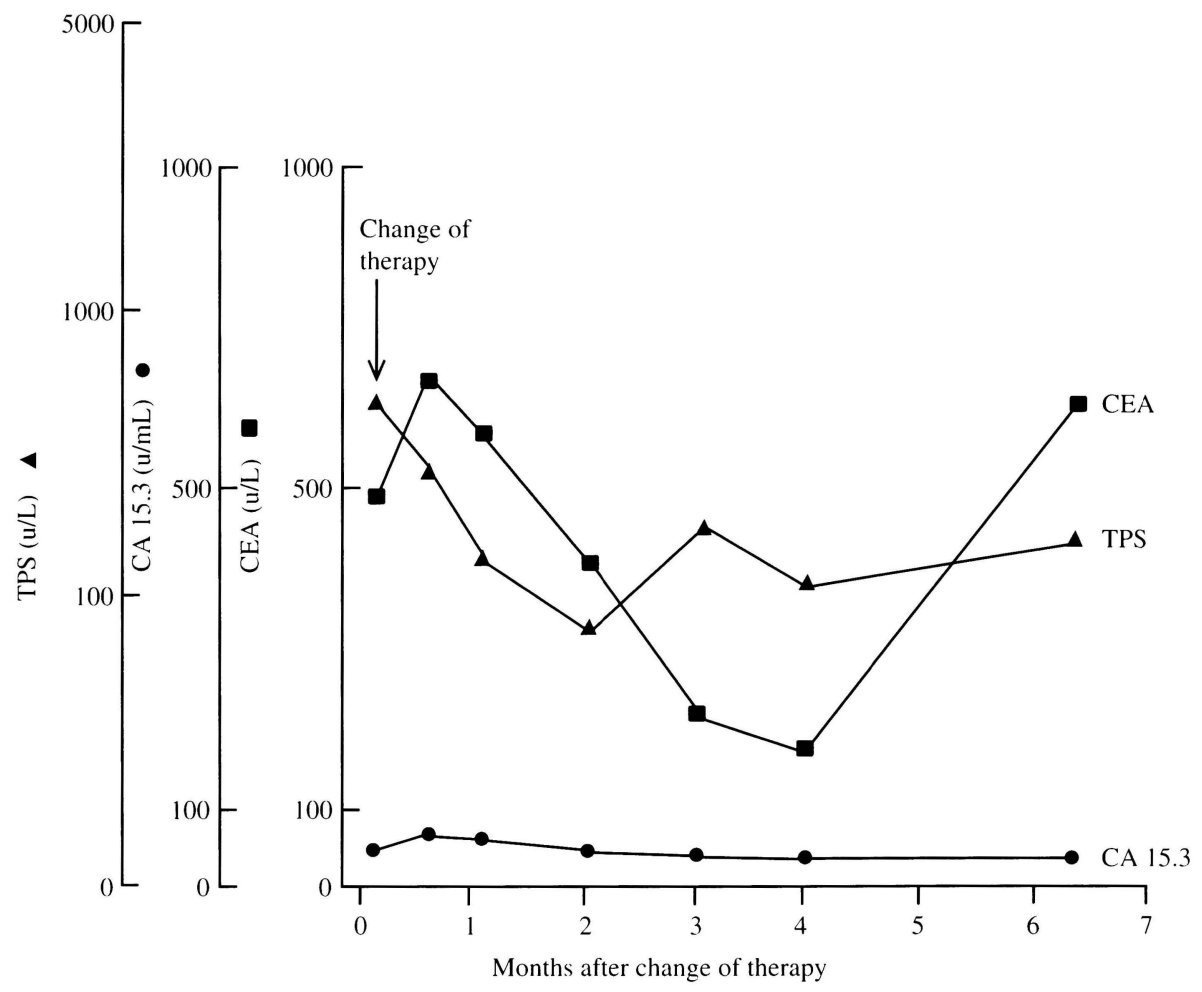

Figure 1. Marker levels for Patient HF, who was in partial remission at 3 months, but had progressive disease at 6 months after change of therapy

with $75 \%$ of CA 15.3 and $67 \%$ of CEA levels, some showing a response for one marker and not another. Likewise, in the "sustained static disease", $60 \%$ of TPS values, $60 \%$ of CA 15.3 and $80 \%$ of CEA values showed persistent elevation with no tendency to fall towards normal. However, it could be said that "sustained partial remission" and "sustained static disease" are both heterogeneous and poorly defined clinical situations. Whether such marker levels predict long-term survival needs to be assessed with further follow-up.

We also found that, in women who at the time of presentation had metastases in visceral organs such as liver, lung or brain alone or in combination, $87 \%$ of TPS values were raised, as compared to $80 \%$ of CA 15.3 and $73 \%$ of CEA values (Table 4). However, in those with bone and soft tissue metastases at presentation, $75 \%$ of TPS values were elevated, compared with $50 \%$ of CA 15.3 and $75 \%$ of CEA values. In addition, we noted that in those with normal TPS marker levels, the sites of subsequent disease progression were limited to bones, regional lymph nodes, skin and soft tissues, though assessment of bone response may not be reliable and provides a poor "gold standard" with which to correlate the marker level.

In conclusion, our small study suggests that TPS provides a more sensitive and specific serological marker than CA 15.3 or CEA for monitoring metastatic breast cancer 
Table 2. Summary of comparison of TPS, CA 15.3 and CEA as early predictors of the effectiveness of therapy.

\begin{tabular}{c|c}
\hline Marker & $\begin{array}{r}\text { No. (\%) of patients with reduction in } \\
\text { markers at } 2-3 \text { weeks after } \\
\text { commencement of effective therapy }\end{array}$ \\
\hline TPS & $9(60 \%)$ \\
CA 15.3 & $4(27 \%)$ \\
\hline
\end{tabular}

Table 3. Comparison of TPS, CA 15.3, and CEA as early detectors of further progression of metastatic disease while on therapy.

\begin{tabular}{c|c}
\hline Marker & $\begin{array}{c}\text { No. }(\%) \text { of patients with elevated or } \\
\text { increasing markers at the time of further } \\
\text { progression of metastatic disease while on therapy }\end{array}$ \\
\hline TPS & $12(86 \%)$ \\
CA 15.3 & $10(71 \%)$ \\
CEA & $5(36 \%)$ \\
\hline
\end{tabular}

Table 4. Elevated marker levels at different sites of metastatic disease at presentation.

\begin{tabular}{l|c|c|c}
\hline \multirow{2}{*}{$\begin{array}{c}\text { Sites of metastases } \\
\text { at presentation }\end{array}$} & \multicolumn{3}{|c}{$\begin{array}{c}\text { No. (\%) of patients with elevated } \\
\text { marker levels }\end{array}$} \\
\cline { 2 - 4 } & TPS & CA 15.3 & CEA \\
\hline Viscera (liver, lung etc.) & $13(87 \%)$ & $12(80 \%)$ & $7(73 \%)$ \\
Bones and soft tissues & $3(75 \%)$ & $2(50 \%)$ & $3(75 \%)$ \\
\hline
\end{tabular}




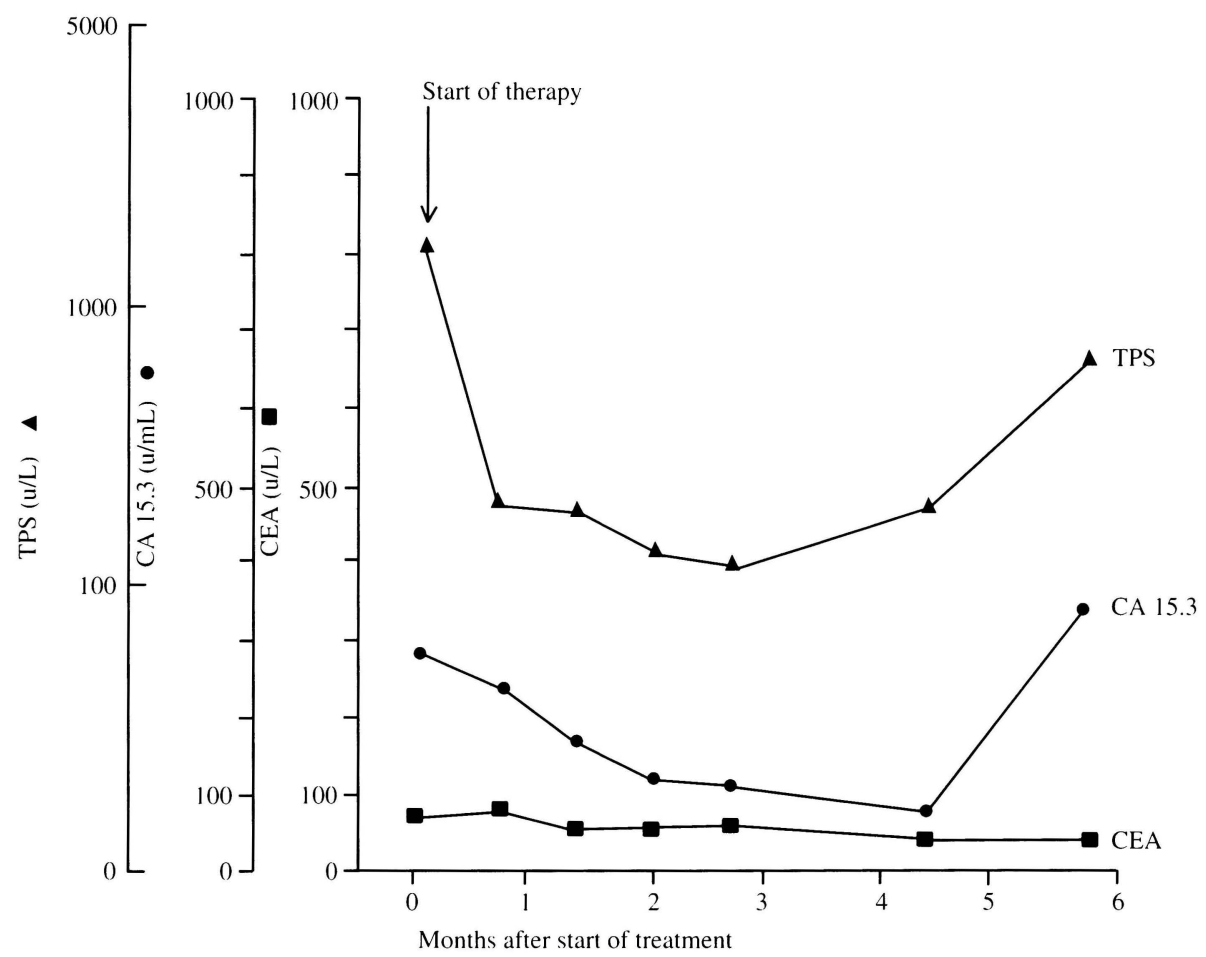

Figure 2. Marker levels of Patient EN, who was in partial remission at 3 months but had progressive disease at 6 months after the start of therapy.

and probably provides improved lead times. Further studies with much larger cohorts of patients will be required to confirm these preliminary results.

It could be argued that none of these markers provides major assistance in the management of metastatic breast cancer, except perhaps in occasionally obviating the need for expensive radiological investigation. However, intervention on the basis of marker trends can be of value in improving the control of advanced breast cancer with chemotherapy. Dixon et al (1991) have shown that patients with falling marker levels will survive longer if chemotherapy is continued, as compared to those patients whose treatment is directed by standard UICC response criteria alone. Use of a novel marker such as TPS, which may be a more sensitive test than those used in Dixon's study, may therefore allow further application of these techniques in future studies.

\section{REFERENCES}

Bieglmayer. C.. Szepesi. T., Neuntenfel, W. (1988). Follow-up of metastatic breast cancer patients w ith a mucin-like carcinoma-associated antigen: Comparison to CA 15.3 and carcinoembryonic antigen. Cancer Lett., 42. 199-205.

Bjorklund. B. (1992). Tumour markers TPA, TPA-S, and cytokeratin. A working hypothesis. Tumordiagn. Ther. 13. 78-80. 
Dixon, A.R., Jonrup, I., Jackson, L., et al. (1991). Serological monitoring of advanced breast cancer treated by systemic cytotoxic using a combination of ESR, CEA and CA 15.3: Fact or fiction? Disease Markers, 9, 167-174.

Haagensen, D.E. Jr (1982). Tumour markers for breast carcinoma. Clin. Lab. Med., 2, 543-564.

Jager, W., Lang, N. (1992). Tumour marker study on breast cancer. Abstract book, 7th International Congress on Serology, 3-7 May, 1992, Rhodes, Erlangen, 1992.

Laurence, D.J.R., Turberville, C., Anderson, S.G. et al. (1975). First British standard for carcinoembryonic antigen (CEA). Br. J. Cancer, 32, 295-299.

Merimsky, O., Inbar, M., Harenvein, M., et al. (1991). Serial serum measurement in the followup of breast cancer patients. Eur. J. Cancer, 27, 1440-1444.

Nicolini, A., Carpi, A., Di Marco, G. et al. (1989). A rational post-operative follow-up with carcinoembryonic antigen, tissue polypeptide antigen and urinary hydroxyproline in breast cancer patients. Cancer, 63, 2037-2046.

Nicolini, A., Colombini, C., Luciani, L. et al. (1991). Evolution of serum CA 15.3 determination with CEA and TPA in the post-operative follow-up of breast cancer patients. Br. J. Cancer, 64, 154-158.

Van Dalen, A. (1992). TPS in breast cancer. A comparative study with carcinoembryonic antigen and CA 15.3. Tumour Biol., 13, 10-17.

Van Dalen, A. et al. (1993). In preparation. 


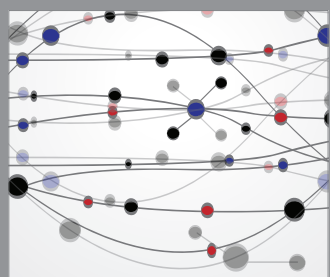

The Scientific World Journal
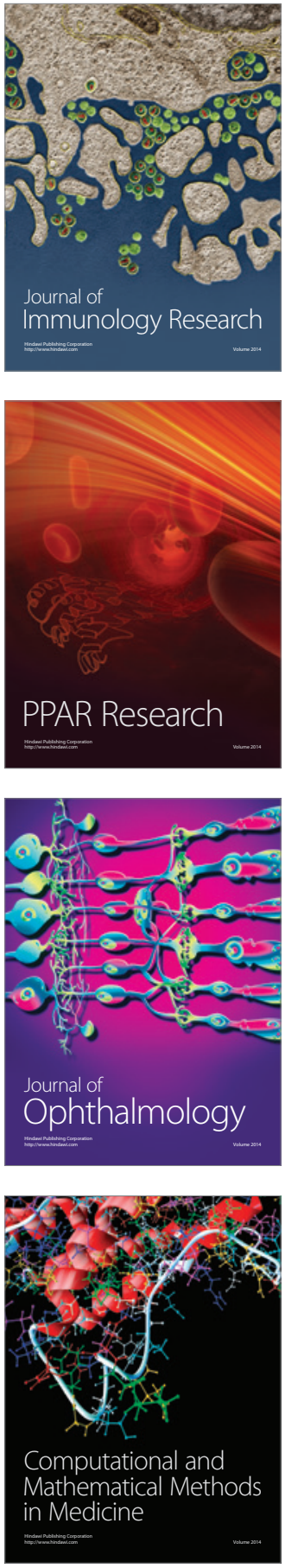

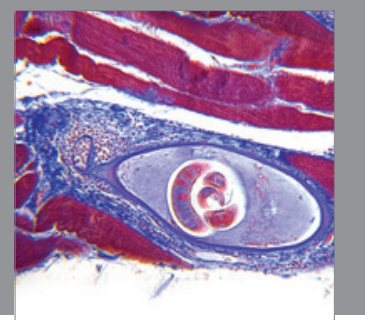

Gastroenterology

Research and Practice
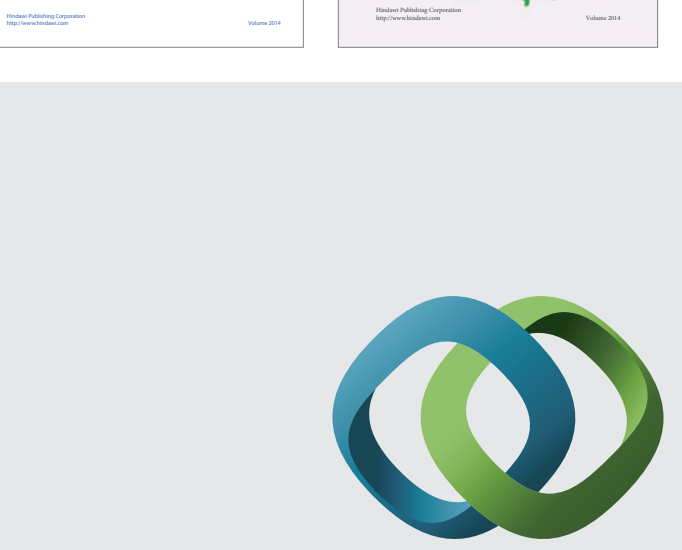

\section{Hindawi}

Submit your manuscripts at

http://www.hindawi.com
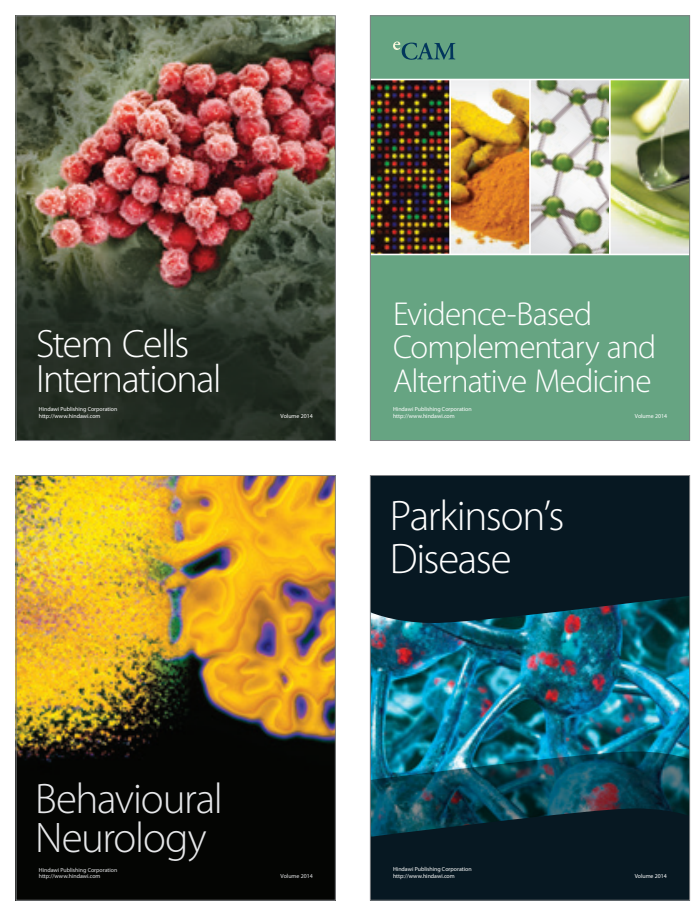

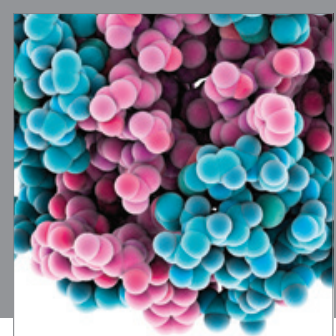

Journal of
Diabetes Research

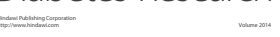

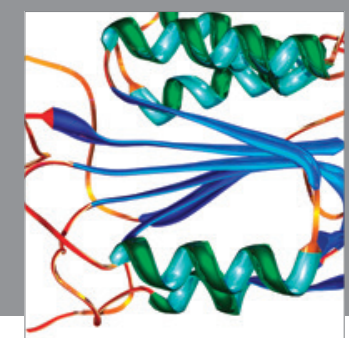

Disease Markers
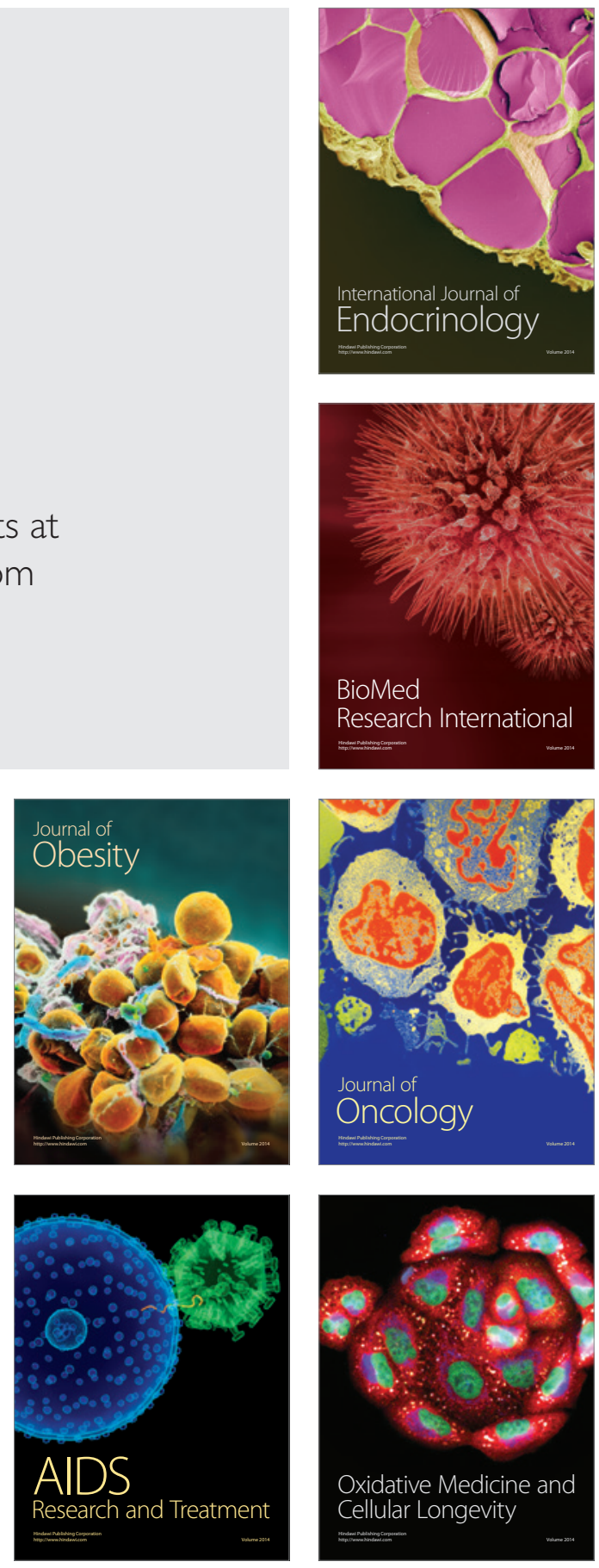